\title{
Characterization of Candida Species from Different Populations in Taiwan
}

\author{
Yun-Liang Yang • Li-Yun Hsieh • \\ An-Huei Wang · Hsiu-Jung Lo • \\ TSARY Hospitals
}

Received: 22 October 2010/Accepted: 17 February 2011/Published online: 3 March 2011

(C) Springer Science+Business Media B.V. 2011

\begin{abstract}
The opportunistic Candida species existing as part of commensal microbiota in humans are usually the etiological agents causing infections. We investigated whether isolates collected from different age groups, hospital units, and sources have distinct characteristics. A total of 913 isolates comprising 395 Candida albicans, 230 Candida tropicalis, 202 Candida glabrata, 62 Candida parapsilosis, 13 Candida krusei, and 11 of other six species were analyzed. Urine was the most common source $(41.2 \%)$, followed by sputum (16.3\%), blood (15.2\%), and others (27.3\%). Candida albicans and C. parapsilosis were more prevalent in the working group [from 19 to 65 years], whereas $C$. tropicalis and $C$. glabrata were more prevalent in the elder one ( $\geq 66$ years). We found that the age of patients and the source of isolates affect the distribution of species. On the other hand, the drug susceptibility of isolates was associated with fungal species and whether patients were hospitalized.
\end{abstract}

Y.-L. Yang

Department of Biological Science and Technology,

National Chiao Tung University, Hsinchu, Taiwan

Y.-L. Yang

Institute of Molecular Medicine and Bioengineering,

National Chiao Tung University, Hsinchu, Taiwan

L.-Y. Hsieh · A.-H. Wang · H.-J. Lo ( $\square)$

Division of Infectious Diseases, National Health Research

Institutes, 35 Keyan Road, Zhunan Town,

Miaoli County, Taiwan, ROC

e-mail: hjlo@nhri.org.tw
Keywords Age $\cdot$ Candida species - Hospital unit . Source $\cdot$ TSARY $\cdot$ Yeast pathogen

\section{Introduction}

Due to the increased size of populations at risk, the prevalence of fungal infections has increased significantly. Candida species are the most frequently isolated fungal pathogens, causing morbidity and mortality in seriously immunocompromised hosts [1-4]. Overall, the five most frequently isolates of Candida species are Candida albicans, Candida tropicalis, Candida glabrata, Candida parapsilosis, and Candida krusei.

The opportunistic Candida species existing as parts of commensal microbiota in humans are usually the etiological agents causing infections [5-8]. Thus, it is important to investigate whether isolates from various sources and various patients with different ages and in different hospital units have distinct characteristics. However, most available epidemiological data were related to candidemia [1-4, 9]. Thus, a national survey, Taiwan Surveillance of Antimicrobial Resistance of Yeasts (TSARY), was initiated in 1999 to analyze yeasts isolated from different sources [10]. In the present study, a continuous study of TSARY 2006 [11] was conducted to investigate whether isolates from sterile sites vs. non-sterile ones and hospitalized patients vs. outpatients have distinct characteristics. Furthermore, 
the distribution and drug susceptibility of Candida species from different age groups, hospital units, and sources were also analyzed.

\section{Materials and Methods}

\section{Organisms and Media}

Yeast isolates were collected in the previous study from the 22 hospitals participating in TSARY 2006 [11]. Each hospital was asked to document total number of yeast isolates isolated from July to September in 2006. For further analysis, all yeast pathogens from sterile sites and up to ten C. albicans and 40 non-albicans Candida species isolates from non-sterile sites were obtained from each hospital. In principle, only one isolate was accepted from each specimen. Nevertheless, when there were multiple species isolated from the same specimen, one isolate from each species was analyzed. After their arrival at the laboratory at National Health Research Institutes (NHRI), these isolates were first sub-cultured on Sabouraud dextrose agar (SDA, BBL, BectonDickinson Cockeysville, MD, USA) to assess the purity and identification. Pure isolates were labeled and stored in vials containing $50 \%$ glycerol at $-70^{\circ} \mathrm{C}$ awaiting further analysis. One hospital did not provide sufficient information. Thus, 913 patients from 21 participating hospitals were analyzed.

\section{Identification}

The identifications of the isolates were reassessed in the laboratory at the NHRI. The identification procedure for the yeast isolates was described in our previous report [11]. All isolates were subjected to analyses with ID 32C (bioMérieux, Marcy l'Etoile, France). The VITEK Yeast Biochemical Card (YBC, bioMérieux, Marcy l'Etoile, France) was then used for the identification of the isolates when the result of ID 32C showed less than $90 \%$ probability and of those with discrepancy from the information provided by the hospitals. The sequences of internal transcribed spacer regions (ITS) and the D1/D2 region of ribosomal DNA [12] were subjected to further assessment when both ID $32 \mathrm{C}$ and YBC failed to reach a probability greater than $90 \%$.
Antifungal Susceptibility Testing

The minimum inhibitory concentrations (MICs) of antifungal agents were determined according to the procedures in our previous studies [13, 14], which followed the guidelines of Clinical and Laboratory Standards Institute [15]. The RPMI medium 1640 (Gibco BRL 31800-022) was used for the testing. Strains from American Type Culture Collection, including C. albicans (ATCC 90028), C. krusei (ATCC 6258), and C. parapsilosis (ATCC 22019), were used as the standard controls. The final growth of each isolate was measured by Biotrak II plate spectrophotometric reader (Amershan Biosciences, Biochrom Ltd., Cambridge England) after incubation at $35^{\circ} \mathrm{C}$ for 24 and 48 hours (h).

The MICs for amphotericin B and azoles were defined as the lowest concentrations capable of preventing any discernible growth and of reducing the turbidity of cells more than $50 \%$, respectively. For amphotericin B, MICs of $\geq 2 \mu \mathrm{g} / \mathrm{ml}$ were considered resistant and $\leq 1 \mu \mathrm{g} / \mathrm{ml}$ were considered susceptible. Isolates with fluconazole $\mathrm{MICs} \geq 64 \mu \mathrm{g} / \mathrm{ml}$ were considered to be resistant, whereas those $\leq 8 \mu \mathrm{g} / \mathrm{ml}$ were susceptible, and those in the range of $16-32 \mu \mathrm{g} / \mathrm{ml}$ were susceptible dose dependent. Isolates with voriconazole MICs $\geq 4 \mu \mathrm{g} / \mathrm{ml}$ were considered to be resistant, whereas those $\leq 1 \mu \mathrm{g} / \mathrm{ml}$ were susceptible, and those with $2 \mu \mathrm{g} / \mathrm{ml}$ was susceptible dose dependent.

Among the phenomena associated with drug resistance, "trailing" describes the reduced but persistent growth that some isolates exhibit at drug concentrations above the MIC in broth dilution tests with azole antifungal agents, such as fluconazole [16]. When the MIC of an isolate after 48-h incubation is approximately fourfold higher than that at the 24-h point, the isolate is defined to have trailing growth $[11,17]$.

\section{Database and Analysis}

The database for this study contained the characteristic information of each submitted isolate. The patients were divided into three groups according to our previous criterion [18], including younger group with age $\leq 18$ years (y), working one with age from 19 to 65 years, and elder one with age $\geq 66$ years. The statistic significance of the differences in frequencies 
and proportions was determined by the chi-square test with Mantel-Haenszel's correction or Fisher exact correction. Logistic regression was applied to assess the independent effect of factors, including age, hospital unit, source, and species.

\section{Results}

\section{The Distribution of Candida Species}

A total of 3,767 yeast isolates were isolated at the 22 hospitals participating in TSARY from July to September in 2006. Among the 3,501 isolates identified as Candida species by the hospitals, C. albicans $(72.3 \%)$ was the most common species, followed by C. tropicalis (12.3\%), C. glabrata (11.7\%), C. parapsilosis $(2.7 \%)$, C. krusei $(0.7 \%)$, and others $(0.3 \%)$. The medical information of 913 isolates was provided by 21 of the hospitals and analyzed in the present study. The average number of isolates contributed per hospital was 43 (ranging from 11 to 80). There were 395 (43.3\%) C. albicans, 230 (25.2\%) C. tropicalis, $202(22.1 \%)$ C. glabrata, 62 (6.8\%) C. parapsilosis, 13 (1.4\%) C. krusei, 4 (0.4\%) Candida lusitaniae, 3 (0.3\%) Candida metapsilosis, and one isolate each of Cryptococcus curvatus, Candida guilliermondii, Candida lipolytica, and Candida rugosa (Table 1).

Since each hospital was asked to submit up to ten C. albicans isolates from non-sterile sites during collection period, the proportion of $C$. albicans was significantly underestimated $(43.3 \%$ in the 913 isolates vs. $72.3 \%$ in the 3,501 isolates). Consequently, the source distribution of this species was not calculated.

As expected, majority of the patients $(83.8 \%$, $P=0.008$ ) were hospitalized when the isolates were recovered. There were 286 and 479 patients in ICU and non-ICU, respectively. A total of 113 and 405 non-albicans Candida species were recovered from sterile and non-sterile sites, respectively. A higher proportion of $C$. tropicalis was from sputum $(P=0.0007)$, whereas the majority of $C$. glabrata in the present study were isolated from urine $(67.8 \%$, $P=0.00002)$. Consequently, significantly less $C$. glabrata were isolated from sputum $(P=0.02)$ and pus $(P=0.01)$. In addition, only $19(13.7 \%)$ candidemia were caused by $C$. glabrata.
The Distribution of Patient Ages

More than half of the isolates were from the elder group, accounting for $58.7 \%$, followed by the working one $(38.2 \%)$ and the younger one $(3.1 \%)$ (Table 2$)$. With the exception of $C$. krusei, the distributions of other species between the working group and the elder one were significantly different. Candida albicans $(P=0.05)$ and $C$. parapsilosis $(P=0.000002)$ were more prevalent in the working group, whereas C. tropicalis $(P=0.04)$ and $C$. glabrata $(P=0.02)$ were more prevalent in the elder one.

The distribution of isolates from urine and blood were different among different age groups. There were $14.3,20.3$, and $11.9 \%$ isolates recovered from the blood of patients with age $\leq 18,19-65$, and $\geq 66$, respectively. There were more isolates from blood in the working group than those in others $(P=0.0008)$. A total of $42.9,26.6$, and $50.6 \%$ of isolates from patients with age $\leq 18,19-65$, and $\geq 66$, respectively, were from urine. Furthermore, nine of the ten isolates $(P=0.05)$ from ear and all nine isolates from genital tract $(P=0.02)$ were recovered from the working group. A total of $89.3,74.5$, and $89.6 \%$ of patients with age $\leq 18,19-65$, and $\geq 66$ were hospitalized, respectively. As expected, the working group had a lower rate of hospitalization than others $(P<0.000001)$.

\section{The Distribution of Sources}

Of all sources, urine was the most common one, accounting for $41.2 \%$ of total isolates, followed by sputum $(16.3 \%)$, blood $(15.2 \%)$, wound $(4.7 \%)$, pus (4.3\%), ascites (4.2\%), tip (3.2\%), catheter (1.6\%), ear $(1.1 \%)$, genital tract $(1 \%)$, and others $(6.4 \%)$. There were $267(29.2 \%)$ isolates recovered from 17 sterile sites, and blood was the most common source $(52.1 \%)$ followed by pus $(14.6 \%)$, ascites $(14.2 \%)$, catheter (5.6\%), and others $(13.5 \%)$ (Table 3). Among the 20 non-sterile sites contributing 646 isolates, urine was the most common source (58.2\%) followed by sputum $(23.1 \%)$, wound $(6.7 \%)$, and others $(12 \%)$ (Table 3$)$.

The distribution of sources were different between outpatients and hospitalized patients. There were more isolates from sterile sites in hospitalized patients than those in outpatients $(P=0.05)$, especially for blood $(P=0.002)$. Nevertheless, there were more isolates from sputum $(P=0.0006)$ in hospitalized 
Table 1 Characteristics of 913 isolate by species

\begin{tabular}{|c|c|c|c|c|c|c|c|}
\hline & C. albicans & C. tropicalis & C. glabrata & C. parapsilosis & C. krusei & Others & Total \\
\hline Total & 395 & 230 & 202 & 62 & 13 & 11 & 913 \\
\hline \multicolumn{8}{|l|}{ Source } \\
\hline \multicolumn{8}{|l|}{ Sterile } \\
\hline Blood & 80 & $29(12.6)^{\mathrm{a}}$ & $19(9.4)$ & $9(14.5)$ & $1(7.7)$ & $1(9.1)$ & 139 (15.2) \\
\hline Pus & 20 & $8(3.5)$ & $2(1)^{b}$ & $6(9.7)^{b}$ & $2(15.4)^{\mathrm{b}}$ & $1(9.1)$ & $39(4.3)$ \\
\hline Ascites & 24 & $7(3)$ & $4(2)$ & $2(3.2)$ & $1(7.7)$ & 0 & $38(4.2)$ \\
\hline Catheter & 7 & $5(2.2)$ & $1(0.5)$ & $2(3.2)$ & 0 & 0 & 15 (1.6) \\
\hline Bile & 7 & 0 & $1(0.5)$ & 0 & 0 & 0 & $8(0.9)$ \\
\hline Others & 16 & $4(1.7)$ & $4(2)$ & $4(6.5)$ & 0 & 0 & $28(3.1)$ \\
\hline Subtotal & 154 & $53(23)$ & $31(15.3)$ & $23(37.1)^{\mathrm{b}}$ & $4(30.8)$ & $2(18.2)$ & $267(29.2)$ \\
\hline \multicolumn{8}{|l|}{ Non-sterile } \\
\hline Urine & 118 & $99(43)$ & $137(67.8)^{b}$ & $13(21)^{\mathrm{b}}$ & $5(38.5)$ & $4(36.4)$ & $376(41.2)$ \\
\hline Sputum & 63 & $63(27.4)^{\mathrm{b}}$ & $19(9.4)^{\mathrm{b}}$ & $2(3.2)^{\mathrm{b}}$ & $2(15.4)$ & 0 & 149 (16.3) \\
\hline Wound & 21 & $6(2.6)$ & $6(3)$ & $5(8.1)$ & $1(7.7)$ & $4(36.4)$ & $43(4.7)$ \\
\hline Tip & 15 & $8(3.5)$ & $3(1.5)$ & $3(4.8)$ & 0 & 0 & $29(3.2)$ \\
\hline Ear & 2 & 0 & 0 & $8(12.9)^{\mathrm{b}}$ & 0 & 0 & $10(1.1)$ \\
\hline Genital tract & 6 & 0 & $3(1.5)$ & 0 & 0 & 0 & $9(1)$ \\
\hline Others & 16 & $1(0.4)$ & $3(1.5)$ & $8(12.9)$ & $1(7.7)$ & $1(9.1)$ & $30(3.3)$ \\
\hline Subtotal & 241 & 177 (77) & $171(84.7)$ & $39(62.9)$ & $9(69.2)$ & $9(81.8)$ & $646(70.8)$ \\
\hline \multicolumn{8}{|l|}{$\operatorname{MICs}(\mu \mathrm{g} / \mathrm{ml})$} \\
\hline Amp $48 \mathrm{~h} \geq 2$ & $1(0.3)$ & $11(4.8)$ & $1(0.5)$ & 0 & $2(15.4)$ & $1(9)$ & $16(1.8)$ \\
\hline Flu $24 \mathrm{~h} \geq 64$ & $10(2.5)^{b}$ & $40(17.4)^{\mathrm{b}}$ & $5(2.5)^{b}$ & $0^{\mathrm{b}}$ & $5(38.5)^{b}$ & 0 & $60(6.6)$ \\
\hline Flu $48 \mathrm{~h} \geq 64$ & $10(2.5)^{b}$ & $123(53.5)^{\mathrm{b}}$ & $7(3.5)^{b}$ & $0^{\mathrm{b}}$ & $13(100)^{\mathrm{b}}$ & 0 & $153(16.8)$ \\
\hline Vor $24 \mathrm{~h} \geq 4(N=911)^{\mathrm{c}}$ & $9(2.3)^{b}$ & $39(17)^{\mathrm{b}}$ & $4(2)^{\mathrm{b}}$ & 0 & 0 & 0 & $52(5.7)$ \\
\hline Vor $48 \mathrm{~h} \geq 4$ & $14(3.5)^{\mathrm{b}}$ & $124(53.9)^{\mathrm{b}}$ & $6(3)^{b}$ & $0^{\mathrm{b}}$ & 0 & 0 & 144 (15.8) \\
\hline \multicolumn{8}{|l|}{ Unit } \\
\hline $\mathrm{ICU}$ & 120 & 85 & 60 & 14 & 6 & 1 & 286 \\
\hline Flu $24 \mathrm{~h} \geq 64$ & $4(1.4)$ & $14(4.9)$ & $2(0.7)$ & 0 & $2(0.7)$ & 0 & $22(7.7)$ \\
\hline Vor $24 \mathrm{~h} \geq 4$ & $2(1.7)$ & $16(18.8)$ & $2(3.3)$ & 0 & 0 & 0 & $20(7)$ \\
\hline Non-ICU & 217 & 123 & 102 & 23 & 5 & 9 & 479 \\
\hline Flu $24 \mathrm{~h} \geq 64$ & $4(0.8)$ & $23(4.8)$ & $2(0.4)$ & 0 & $3(0.6)$ & 0 & $32(6.7)$ \\
\hline Vor $24 \mathrm{~h} \geq 4$ & $5(2.3)$ & $21(17.1)$ & $2(3.3)$ & 0 & 0 & 0 & $28(5.8)$ \\
\hline Outpatient & 58 & 22 & 40 & 25 & 2 & 1 & 148 \\
\hline Flu $24 \mathrm{~h} \geq 64$ & $2(1.4)$ & $3(2)$ & $1(0.7)$ & 0 & 0 & 0 & $6(4.1)$ \\
\hline Vor $24 \mathrm{~h} \geq 4(N=146)^{\mathrm{c}}$ & $2(3.4)$ & $2(9.1)$ & 0 & 0 & 0 & 0 & $4(2.7)$ \\
\hline
\end{tabular}

Amp amphotericin B, Flu fluconazole, ICU intensive care unit, MIC minimum inhibitory concentration, Vor voriconazole

${ }^{\text {a }}$ Number of isolate (percentage in the same species group)

b $P \leq 0.05$, comparison of percentage for each characteristic/species group vs. percentage in total population

${ }^{\text {c }}$ MICs of one of each C. glabrata and C. parapsilosis from outpatients grew too slowly to be determined

patients than those in outpatients. Furthermore, all 27 isolates from tip were from hospitalized patients and there were more isolates from ear or genital tract in outpatients than those in hospitalized patients
$(P<0.000001)$. It is not surprising that more nonsterile site isolates were recovered from outpatients since they were relatively less ill and few to have blood drawn for culture. 
Table 2 Characteristics of 913 isolates by age group of patients
Amp amphotericin B, Flu fluconazole, $I C U$ intensive care unit, $M I C$ minimum inhibitory concentration, Vor voriconazole

a Number of isolate (percentage in the same age group)

b $P \leq 0.05$, comparison of percentage for each characteristic/age group vs. percentage in total population

c MICs of one of each $19-65$ years and $\geq 66$ years grew too slowly to be determined

\begin{tabular}{|c|c|c|c|c|}
\hline & $\leq 18$ years & 19-65 years & $\geq 66$ years & Total \\
\hline Total & 28 & 349 & 536 & 913 \\
\hline \multicolumn{5}{|l|}{ Species } \\
\hline C. albicans & $15(53.6)^{\mathrm{a}}$ & $164(47)$ & $216(40.3)$ & $395(43.3)$ \\
\hline C. tropicalis & $4(14.3)$ & $76(21.8)$ & $150(28)$ & $230(25.2)$ \\
\hline C. glabrata & $3(10.7)$ & $63(18.1)$ & $136(25.4)$ & $202(22.1)$ \\
\hline C. parapsilosis & $3(10.7)$ & $41(11.7)^{\mathrm{b}}$ & $18(3.4)^{\mathrm{b}}$ & $62(6.8)$ \\
\hline C. krusei & $1(3.6)$ & $3(0.9)$ & $9(1.7)$ & $13(1.4)$ \\
\hline Others & $2(7.1)$ & $2(0.6)$ & $7(1.3)$ & $11(1.2)$ \\
\hline \multicolumn{5}{|l|}{ Source } \\
\hline \multicolumn{5}{|l|}{ Sterile } \\
\hline Blood & $4(14.3)$ & $71(20.3)^{b}$ & 64 (11.9) & 139 (15.2) \\
\hline Pus & $2(7.1)$ & $22(6.3)$ & $15(2.8)$ & $39(4.3)$ \\
\hline Ascites & $1(3.6)$ & $20(5.7)$ & $17(3.2)$ & $38(4.2)$ \\
\hline Catheter & $1(3.6)$ & $4(1.1)$ & $10(1.9)$ & 15 (1.6) \\
\hline Bile & 0 & $3(0.9)$ & $5(0.9)$ & $8(0.9)$ \\
\hline Others & $4(14.3)$ & $14(4)$ & $10(1.9)$ & $28(3.1)$ \\
\hline Subtotal & $12(42.9)$ & $134(38.4)^{\mathrm{b}}$ & $121(22.6)^{\mathrm{b}}$ & $267(29.2)$ \\
\hline \multicolumn{5}{|l|}{ Non-sterile } \\
\hline Urine & 12 (42.9) & $93(26.6)^{b}$ & $271(50.6)^{b}$ & $376(41.2)$ \\
\hline Sputum & $3(10.7)$ & 42 (12) & 104 (19.4) & 149 (16.3) \\
\hline Wound & 0 & $23(6.6)$ & $20(3.7)$ & $43(4.7)$ \\
\hline Tip & 1 (3.6) & $13(3.7)$ & $15(2.8)$ & $29(3.2)$ \\
\hline Ear & 0 & $9(2.6)$ & $1(0.2)^{b}$ & $10(1.1)$ \\
\hline Genital tract & 0 & $9(2.6)$ & $0^{\mathrm{b}}$ & $9(1)$ \\
\hline Others & 0 & $26(7.4)$ & $4(0.7)$ & $30(3.3)$ \\
\hline Subtotal & $16(57.1)$ & $215(61.6)^{\mathrm{b}}$ & $415(77.4)^{\mathrm{b}}$ & $646(70.8)$ \\
\hline \multicolumn{5}{|l|}{ Unit } \\
\hline $\mathrm{ICU}$ & $12(42.9)$ & $65(18.6)^{b}$ & $209(39)^{\mathrm{b}}$ & $286(31.3)$ \\
\hline Non-ICU & $13(46.4)$ & $195(55.9)$ & $271(50.6)$ & $479(52.5)$ \\
\hline Outpatient & $3(10.7)$ & $89(25.5)^{b}$ & $56(10.4)^{\mathrm{b}}$ & $148(16.2)$ \\
\hline \multicolumn{5}{|l|}{$\operatorname{MICs}(\mu \mathrm{g} / \mathrm{ml})$ at $24 \mathrm{~h}$} \\
\hline Flu $\geq 64$ & $1(3.6)$ & $25(7.2)$ & $34(6.3)$ & $60(6.6)$ \\
\hline Vor $\geq 4(\mathrm{~N}=911)^{\mathrm{c}}$ & $1(3.6)$ & $20(5.7)$ & $31(5.8)$ & $52(5.7)$ \\
\hline
\end{tabular}

\section{Antifungal Susceptibilities of Yeasts}

After 48-h incubation, the overall ratio of resistant isolates to amphotericin $\mathrm{B}$ was $1.8 \%$. The 16 amphotericin B-resistant isolates were consisted of 11 C. tropicalis, 2 C. krusei, and one isolate each of C. albicans, C. glabrata, and C. curvatus (Table 1): a total of 2, 6, and 8 isolates from patients with age $\leq 18,19-65$, and $\geq 66$, respectively (Table 2 ).

Of the 153 isolates, comprising 123 C. tropicalis, 13 C. krusei, 10 C. albicans, and 7 C. glabrata, having fluconazole MICs $\geq 64 \mu \mathrm{g} / \mathrm{ml}$ after 48 -h incubation, 98 (64\%) showed trailing phenotype. They included 87 C. tropicalis, 7 C. albicans, and 4 C. glabrata. The issue of trailing is problematic for interpreting the results of fluconazole MICs at $48 \mathrm{~h}$. Therefore, in the present study, we analyzed whether there were any characteristics associated with isolates having fluconazole MICs $\geq 64 \mu \mathrm{g} / \mathrm{ml}$ after 24-h incubation, considered as resistant. The percentage of fluconazole-resistant isolates was not different among different age populations (Table 2). The proportion 
Table 3 Characteristics of 913 isolates by sources

\begin{tabular}{|c|c|c|c|c|c|c|}
\hline & \multicolumn{3}{|l|}{ Unit } & \multirow[b]{2}{*}{ Total } & \multicolumn{2}{|c|}{ MICs at $24 \mathrm{~h}(\mu \mathrm{g} / \mathrm{ml})$} \\
\hline & ICU & Non-ICU & Outpatient & & Flu $\geq 64$ & Vor $\geq 4(N=911)^{\mathrm{c}}$ \\
\hline Total & 286 & 479 & 148 & 913 & $60(6.6)$ & $52(5.7)$ \\
\hline \multicolumn{7}{|l|}{ Sterile } \\
\hline Blood & $45(32.4)^{\mathrm{a}}$ & $84(60.4)$ & $10(7.2)^{\mathrm{b}}$ & 139 & $8(5.8)$ & $7(5)$ \\
\hline Pus & $6(15.4)$ & $22(56.4))$ & $11(28.2)$ & 39 & 0 & 0 \\
\hline Ascites & $16(42.1)$ & $18(47.4)$ & $4(10.5)$ & 38 & $1(2.6)$ & 0 \\
\hline Catheter & $7(46.7)$ & $6(40)$ & $2(13.3)$ & 15 & $3(20)$ & $2(13.3)$ \\
\hline Bile & $1(12.5)$ & $6(75)$ & $1(12.5)$ & 8 & 0 & 0 \\
\hline Others & $7(25)$ & $16(57.1)$ & $5(17.9)$ & 28 & $2(7.2)$ & $1(3.6)$ \\
\hline Subtotal & $82(30.7)$ & $152(56.9)$ & 33 (12.4) & 267 & $14(5.2)$ & $10(3.8)$ \\
\hline \multicolumn{7}{|l|}{ Non-sterile } \\
\hline Urine & $130(34.6)$ & $184(48.9)$ & $62(16.5)$ & 376 & $26(6.9)$ & $20(5.3)^{\mathrm{c}}$ \\
\hline Sputum & $57(38.3)$ & $82(55)$ & $10(6.7)^{\mathrm{b}}$ & 149 & $15(10.1)$ & $16(10.7)$ \\
\hline Wound & $6(14)$ & $31(72)$ & $6(14)$ & 43 & $2(4.7)$ & $2(4.7)$ \\
\hline Tip & $10(34.5)$ & $19(65.5)$ & $0^{\mathrm{b}}$ & 29 & 0 & $2(6.9)$ \\
\hline Ear & 0 & $1(10)$ & $9(90)^{\mathrm{b}}$ & 10 & 0 & 0 \\
\hline Genital tract & 0 & $0^{\mathrm{b}}$ & $9(100)^{b}$ & 9 & $1(11.1)$ & $1(11.1)$ \\
\hline Others & $1(3.3)$ & $10(33.3)$ & 19 (63.4) & 30 & $2(6.7)$ & $1(3.3)$ \\
\hline Subtotal & 204 (31.6) & $327(50.6)$ & $115(17.8)$ & 646 & $46(7.1)$ & $42(6.5)$ \\
\hline
\end{tabular}

Amp amphotericin B, Flu fluconazole, ICU intensive care unit, $M I C$ minimum inhibitory concentration, Vor voriconazole

${ }^{\text {a }}$ Number of isolate (percentage in the same source group)

b $P \leq 0.05$, comparison of percentage for each characteristic/source group vs. percentage in total population

${ }^{c}$ MICs of one of each isolate from pus and urine grew too slowly to be determined

of the fluconazole-resistant isolates from hospitalized patients was higher than that from outpatients (54/765 vs. $6 / 148, P=0.05$ ) (Table 1 ). As expected, different species had different percentage of fluconazoleresistant isolates. The species of C. krusei (38.5\%) had the highest rate to be fluconazole resistant, followed by C. tropicalis (17.4\%) and C. glabrata (2.5\%). All C. parapsilosis were susceptible to fluconazole. Based on multivariate analysis, C. tropicalis was the only independent risk factor associated with fluconazole resistance (odds ratio, 7.85; 95\% confidence interval, 13.8-16.2).

Trailing phenotype was also observed for voriconazole susceptibility. One $C$. glabrata isolate had voriconazole MICs $\geq 4 \mu \mathrm{g} / \mathrm{ml}$ after 48 -h incubation, whereas the MIC after 24-h incubation was not detected due to slow growth. Of the remaining 143 isolates comprising 124 C. tropicalis, 14 C. albicans, and 5 C. glabrata, having voriconazole MICs $\geq 4 \mu \mathrm{g} / \mathrm{ml}$ after 48-h incubation, 101 (70.6\%) showed trailing phenotype. They included 89 C. tropicalis, 10 C. albicans, and 2 C. glabrata. Again, we analyzed whether there were any characteristics associated with isolates having voriconazole MICs $\geq 4 \mu \mathrm{g} / \mathrm{ml}$ after 24-h incubation, considered as resistant. The percentage of voriconazole-resistant isolates was not different among different age populations (Table 2). Unlike susceptibility to fluconazole, the proportion of the voriconazole-resistant isolates from hospitalized patients was not significantly different from that from outpatients (48/765 vs. $4 / 146, \quad P=0.1$ ) (Table 1). Furthermore, different species had different percentage of voriconazoleresistant isolates. The species of $C$. tropicalis $(17 \%)$ had the highest rate to be voriconazole resistant, followed by C. albicans (2.3\%) and C. glabrata (2\%).

\section{Discussion}

In the past, nearly $80 \%$ of candidemia could be attributed to C. albicans [19]. Although C. albicans is still the most common single species causing 
candidemia in our study $(57.6 \%)$ as well as other studies [1, 3, 9, 20], prevalence of non-C. albicans Candida has indeed increased. Consistent with previous observation that $C$. glabrata is the most common cause of candiduria [13, 14, 21], the majority of this species in the present study were isolated from urine. Even though this species is the second most common cause of candidemia in the USA and the Netherlands [3, 22], only 19 (13.7\%) candidemia were caused by $C$. glabrata in the present study. Even though isolates sent to NHRI had clinical significance, we still cannot rule out the possibility that isolates from non-sterile sites are colonizers. Candida species colonizing in human are usually the causes of infections when patients become immunocompromised. Thus, it is important to investigate isolates from non-sterile sites as well as those from sterile ones for prevention and control strategies.

In our previous report, 58 C. parapsilosis isolates were collected in 2002 and blood was the most common source, accounting for $36.2 \%$, followed by wound $(20.7 \%)$ [18]. In the present study, urine became the most common source, accounting for $21 \%$ of the 62 recovered isolates, followed by blood $(14,5 \%)$, ear $(12.9 \%)$, pus $(9.7 \%)$, and wound $(8.1 \%)$. One explanation for the different source distribution of $C$. parapsilosis from other species is that it can be part of the normal flora on the human skin [23] and has a wide distribution in environments [24, 25]. Nevertheless, factors contributed to the different source distribution of this species between these two studies need further investigation. Among the 515 isolates from either sputum or urine, $375(72.8 \%)$ were from the elder group. We found that the majority of C. glabrata (114/136, 83.8\%) and C. tropicalis $(122 / 150,81.3 \%)$ from the elder group were isolated from either sputum or urine. This observation may explain why both $C$. glabrata and C. tropicalis are more prevalent in elderly patients in the present study. Whether the elderly patients are more susceptible to $C$. glabrata and C. tropicalis infections needs further investigation.

Overexpression of fluconazole transporters and the modification of the target enzymes are the two major mechanisms contributing to fluconazole resistance $[26,27]$. It has been shown that multiple molecular mechanisms contribute to a stepwise development of fluconazole resistance in C. albicans [28]. Even though isolates have trailing phenotype and are not resistant to fluconazole yet, we still cannot ignore those isolates since they are prone to be resistant. The observation that only $2.5 \%$ of $C$. glabrata having fluconazole MICs $\geq 64 \mu \mathrm{g} / \mathrm{ml}$ after 24-h incubation is different from the results in European countries and the United States. One explanation is that in Taiwan, physicians do not administer fungal prophylaxis as frequently as those in other regions [29-31]. Candida glabrata is much more ready to display variation in phenotypes contributing to drug resistance partially due to its haploid nature [32]. Hence, we do not suggest physicians to administer fluconazole therapy for infections caused by $C$. glabrata, even though very few isolates of this species recovered in the present study having fluconazole MICs $\geq 64 \mu \mathrm{g} / \mathrm{ml}$. Furthermore, a correlation of susceptibility to fluconazole and voriconazole was observed in the present study as described in our previous study [33]. Interestingly, no C. krusei was resistant to voriconazole, despite that it is intrinsically resistant to fluconazole.

In conclusion, we have analyzed the species, culture source (sterile, non-sterile), patient location (ICU, non-ICU, outpatient), patient age group, and amphotericin B/fluconazole/voriconazole susceptibility for Candida isolates. We found that the age of patients is associated with species distribution. So is the source of isolates. Furthermore, species and whether the patients were hospitalized are associated with fluconazole susceptibility of the isolates.

Acknowledgments We would like to thank Bristol Myers Squibb and Pfizer for supplying the amphotericin B and fluconazole, respectively. We also thank the 22 participating hospitals for providing clinical isolates and related information. They are Asia East Memorial Hospital, Buddhist Tzu-Chi General Hospital, Chang Gung Memorial Hospital at Kaohsiung, Chiayi Christian Hospital, Chang-Hwa Christian Hospital, Cheng Ching Hospital, Chung Shan Medical Dental College Hospital, Kaohsiung Military Hospital, Kaohsiung Medical College Chung-Ho Memorial Hospital, Kuan-Tien General Hospital, Lo-Hsu Foundation Inc. Lo-Tung Poh Ai Hospital, Miin Sheng General Hospital, National Cheng Kung University Hospital, Show Chwan Memorial Hospital, Sin-Lau Christian Hospital, St. Mary Hospital, Taipei Municipal Chung Hsiao Hospital, Taipei Municipal Hoping Hospital, Veterans General Hospital-Taichung, Veterans General HospitalKaohsiung, Zen Ai General Hospital, and Tungs' Taichung MetroHarbor Hospital, Sha Lu branch. We thank Dr. T. L. Lauderdale, Mr. C. W. Wang, and Ms. W. T. Cheng for their technical assistance. This study was supported by the grant NHRI 99A1-IDPP04-014. The authors declare that they have no competing interests. 


\section{References}

1. Cheng MF, Yu KW, Tang RB, Fan YH, Yang YL, Hsieh KS, et al. Distribution and antifungal susceptibility of Candida species causing candidemia from 1996 to 1999. Diagn Microbiol Infect Dis. 2004;48:33-7.

2. Pfaller MA, Diekema DJ. Epidemiology of invasive candidiasis: a persistent public health problem. Clin Microbiol Rev. 2007;20:133-63.

3. Pfaller MA, Jones RN, Doern GV, Sader HS, Messer SA, Houston A, et al. Bloodstream infections due to Candida species: SENTRY antimicrobial surveillance program in North America and Latin America, 1997-1998. Antimicrob Agents Chemother. 2000;44:747-51.

4. Yang YL, Cheng MF, Wang CW, Wang AH, Cheng WT, Lo HJ, et al. The distribution of species and susceptibility of amphotericin B and fluconazole of yeast pathogens isolated from sterile sites in Taiwan. Med Mycol. 2010;48: 328-34.

5. Chen YC, Chang SC, Tai HM, Hsueh PR, Luh KT. Molecular epidemiology of Candida colonizing critically ill patients in intensive care units. J Formos Med Assoc. 2001;100:791-7.

6. Li SY, Yang YL, Chen KW, Cheng HH, Chiou CS, Wang $\mathrm{TH}$, et al. Molecular epidemiology of long-term colonization of Candida albicans strains from HIV-infected patients. Epidemiol Infect. 2006;134:265-9.

7. Miranda LN, van der Heijden IM, Costa SF, Sousa AP, Sienra RA, Gobara S, et al. Candida colonisation as a source for candidaemia. J Hosp Infect. 2009;72:9-16.

8. Voss A, Hollis RJ, Pfaller MA, Wenzel RP, Doebbeling $\mathrm{BN}$. Investigation of the sequence of colonization and candidemia in nonneutropenic patients. J Clin Microbiol. 1994;32:975-80.

9. Chen PL, Lo HJ, Wu CJ, Lee HC, Chang CM, Lee NY et al. Species distribution and antifungal susceptibility of blood Candida isolates at a tertiary hospital in southern Taiwan, 1999-2006. Mycoses. 2010; In press

10. Lo HJ, Ho AH, Ho M. Factors accounting for mis-identification of Candida species. J Microbiol Immunol Infect. 2001;34:171-7.

11. Yang YL, Wang AH, Wang CW, Cheng WT, Li SY, Lo $\mathrm{HJ}$, et al. Susceptibilities to amphotericin B and fluconazole of Candida species in TSARY 2006. Diagn Microbiol Infect Dis. 2008;61:175-80.

12. Leaw SN, Chang HC, Sun HF, Barton R, Bouchara JP, Chang TC. Identification of medically important yeast species by sequence analysis of the internal transcribed spacer regions. J Clin Microbiol. 2006;44:693-9.

13. Yang YL, Ho YA, Cheng HH, Ho M, Lo HJ. Susceptibilities of Candida species to amphotericin B and fluconazole: the emergence of fluconazole resistance in Candida tropicalis. Infect Control Hosp Epidemiol. 2004; 25:60-4.

14. Yang YL, Li SY, Cheng HH, Lo HJ. Susceptibilities to amphotericin B and fluconazole of Candida species in TSARY 2002. Diagn Microbiol Infect Dis. 2005;51:179-83.

15. Clinical Laboratory Standards Institute CLSI. Reference method for broth dilution antifungal susceptibility testing of yeasts; approved standard-third edition. CLSI document M27-A3. Wayne, PA. 2008
16. Lee MK, Williams LE, Warnock DW, Arthington-Skaggs BA. Drug resistance genes and trailing growth in Candida albicans isolates. J Antimicrob Chemother. 2004;53: 217-24.

17. Arthington-Skaggs BA, Lee-Yang W, Ciblak MA, Frade JP, Brandt ME, Hajjeh RA, et al. Comparison of visual and spectrophotometric methods of broth microdilution MIC end point determination and evaluation of a sterol quantitation method for in vitro susceptibility testing of fluconazole and itraconazole against trailing and nontrailing Candida isolates. Antimicrob Agents Chemother. 2002;46: 2477-81.

18. Yang YL, Cheng HH, Lo HJ. Distribution and antifungal susceptibility of Candida species isolated from different age populations in Taiwan. Med Mycol. 2006;44:237-42.

19. Edwards EJJ. Candida species. In: Mandell GL, Bennett JE, Dolin R, editors. Principles and Practice of Infectious Diseases. 4th ed. New York: Churchill Livingstone Inc; 1995. p. 2289-306.

20. Peman J, Canton E, Gobernado M. Epidemiology and antifungal susceptibility of Candida species isolated from blood: results of a 2-year multicentre study in Spain. Eur J Clin Microbiol Infect Dis. 2005;24:23-30.

21. Pfaller MA, Messer SA, Hollis RJ, Jones RN, Doern GV, Brandt ME, et al. Trends in species distribution and susceptibility to fluconazole among blood stream isolates of Candida species in the United States. Diagn Microbiol Infect Dis. 1999;33:217-22.

22. Voss A, Kluytmans JA, Koeleman JG, Spanjaard L, Vandenbroucke-Grauls CM, Verbrugh HA, et al. Occurrence of yeast bloodstream infections between 1987 and 1995 in five Dutch university hospitals. Eur J Clin Microbiol Infect Dis. 1996;15:909-12.

23. Hedderwick SA, Lyons MJ, Liu M, Vazquez JA, Kauffman CA. Epidemiology of yeast colonization in the intensive care unit. Eur J Clin Microbiol Infect Dis. 2000;19:663-70.

24. Abi-Said D, Anaissie E, Uzun O, Raad I, Pinzcowski H, Vartivarian S. The epidemiology of hematogenous candidiasis caused by different Candida species. Clin Infect Dis. 1997;24:1122-8.

25. Levin AS, Costa SF, Mussi NS, Basso M, Sinto SI, Machado C, et al. Candida parapsilosis fungemia associated with implantable and semi-implantable central venous catheters and the hands of healthcare workers. Diagn Microbiol Infect Dis. 1998;30:243-9.

26. White TC, Marr KA, Bowden RA. Clinical, cellular, and molecular factors that contribute to antifungal drug resistance. Clin Microbiol Rev. 1998;11:382-402.

27. Yang YL, Lo HJ. Mechanisms of antifungal agent resistance. J Microbiol Immunol Infect. 2001;34:79-86.

28. Franz R, Kelly SL, Lamb DC, Kelly DE, Ruhnke M, Morschhäuser J. Multiple molecular mechanisms contribute to a stepwise development of fluconazole resistance in clinical Candida albicans strains. Antimicrob Agents Chemother. 1998;42:3065-72.

29. Cheng MF, Yang YL, Yao TJ, Lin CY, Liu JS, Tang RB. Risk factors for fatal candidemia caused by Candida albicans and non-albicans Candida species. BMC Infect Dis. 2005;5:22.

30. Yang YL, Cheng MF, Chang YW, Young TG, Chi H, Lee $\mathrm{SC}$, et al. Host factors do not influence the colonization or 
infection by fluconazole resistant Candida species in hospitalized patients. J Negat Results Biomed. 2008;7:12.

31. Yang YL, Hung CC, Wang AH, Tseng FC, Leaw SN, Tseng YT, et al. Oropharyngeal colonization of HIVinfected outpatients in Taiwan by yeast pathogens. J Clin Microbiol. 2010;48:2609-12.

32. Bennett JE, Izumikawa K, Marr KA. Mechanism of increased fluconazole resistance in Candida glabrata during prophylaxis. Antimicrob Agents Chemother. 2004; 48:1773-7.

33. Yang YL, Cheng HH, Lo HJ. In vitro activity of voriconazole against Candida species isolated in Taiwan. Int $\mathbf{J}$ Antimicrob Agents. 2004;24:294-6. 